\title{
Monitoring and visualising a neighbourhood area sub-grid
}

DOI:

10.5220/0004409101270131

Link to publication record in Manchester Research Explorer

\section{Citation for published version (APA):}

Pourmirza, Z., \& Brooke, J. M. (2013). Monitoring and visualising a neighbourhood area sub-grid. In SMARTGREENS 2013 - Proceedings of the 2nd International Conference on Smart Grids and Green IT Systems/SMARTGREENS - Proc. Int. Conf. Smart Grids Green IT Syst. (pp. 127-131). Science and Technology Publications Lda. https://doi.org/10.5220/0004409101270131

\section{Published in:}

SMARTGREENS 2013 - Proceedings of the 2nd International Conference on Smart Grids and Green IT Systems|SMARTGREENS - Proc. Int. Conf. Smart Grids Green IT Syst.

\section{Citing this paper}

Please note that where the full-text provided on Manchester Research Explorer is the Author Accepted Manuscript or Proof version this may differ from the final Published version. If citing, it is advised that you check and use the publisher's definitive version.

\section{General rights}

Copyright and moral rights for the publications made accessible in the Research Explorer are retained by the authors and/or other copyright owners and it is a condition of accessing publications that users recognise and abide by the legal requirements associated with these rights.

\section{Takedown policy}

If you believe that this document breaches copyright please refer to the University of Manchester's Takedown Procedures [http://man.ac.uk/04Y6Bo] or contact uml.scholarlycommunications@manchester.ac.uk providing relevant details, so we can investigate your claim.

\section{OPEN ACCESS}




\title{
Monitoring and Visualising a Neighbourhood Area Sub-Grid
}

\author{
Zoya Pourmirza and John M. Brooke \\ School of Computer Science, The University of Manchester, Oxford Road, Manchester, U.K.
}

Keywords: Smart Grid, Communication Network, Neighbourhood Area, Monitoring, Sensor Network, Software Architecture, Visualisation Tool.

\begin{abstract}
In this paper we analyse the architecture and technologies for monitoring the Neighbourhood Area Network (NAN) of the Smart Grid. We consider the role of sensor networks in providing information about the environment of the NAN, for example to monitor temperature and movement of vehicles and people, which can provide useful information about changes in the loading of the NAN. The two main contributions of this research are as follows. Firstly, we develop a software architecture for an ICT network of the Smart Grid which could integrate information from sensors from various levels of the grid. Currently no such architecture has been implemented for collecting data and providing the basis of Decision Support Tools (DSTs) for the NAN level of the grid. Secondly, we have developed a visualisation interface for the human operator of the grid, as the basis for such DSTs, which overlays the information from the sensors and the measurements of the electrical performance of the NAN on a GIS-based view of the NAN. We describe an actual implementation of this design currently being installed in the sub-Grid supplying the University of Manchester which is of comparable size and complexity to urban NANs.
\end{abstract}

\section{INTRODUCTION}

The intelligent electrical networks called Smart Gids incorporate communcations and information technology to service the generation, transmission and finally the distribution networks of the power grid. To reflect this structure the ICT network of the Smart Grid is divided into three networks. These networks can be considered as the Wide Area Network (WAN), Neighbourhood Area Network (NAN) and Home Area Network (HAN). The lack of research on the monitoring and predictive realtime system control in the NAN leads us to focus on this specific level of the Smart Grid.

We have designed and implemented a software architecture for the ICT in the NAN. It integrates data from smart meters for controlling building data, wireless sensors for monitoring the environment and devices which measure the electrical behaviour of the power network. Finally, based on our software architecture, a visualisation tool has been developed which provides a basis for Decision Support Tools (DSTs) that can be used to plan and operate the Grid at the NAN level.

The remainder of this paper is organised as follows: Section 2 discusses about the usage of the sensor network in the Smart Grid. Section 3 proposes a software architecture for the ICT section of the NAN. Section 4 presents a visualization tool for the NAN. Finally section 5 summarizes our contributions and presents ideas for future work.

\section{THE ROLE OF SENSORNETS}

Power Grids have historically been centrally controlled, with the NAN and HAN levels being essentially passive. Detailed monitoring at this level has therefore not been a priority. As the Grid evolves to a higher degree of localised control, the types of sensors and computational units should become more lightweight and widely deployed. We gather two types of real-time information, firstly measurements of the electric network itself and secondly measurements of the environment of the NAN. The first type of information enables the detection of abnormal behaviour which can identify faulty components before their failure leads to more widespread failures of the system. The second type enables predictions of future demand to be made based on environmental variables that influence local demand. 
We choose to monitor the NAN by a WSN. We selected TinyOS (Levis et al., 2005) sensors as a prototype to evaluate the architectural proposal by simulation. Accordingly, TOSSIM (Levis and Lee, 2003) the WSN simulator for the TinyOS sensors has been selected to simulate the network. TOSSIM has some advantages over the other WSN simulators which will be discussed later. A range of applications of the WSN in Distribution Networks (of which NANs are an example) are identified in (Pourmirza and Brooke, 2012b). The ones we are particularly interested in are local weather condition monitoring and lighting in order to find the relation between these parameters and electricity consumption.

\section{MONITORING A SUB-GRID ON A UNIVERSITY CAMPUS}

In designing an ICT architecture for the NAN subGrid, we choose to componentize the NAN system into interacting sub-systems. This architecture has various advantages such as preventing single point of failure, dealing with a potential information flood caused by the centralized system, and applying finer grained monitoring and control at the level that was blind previously. Additionally, utilising cluster based communication and componentizing the ICT network monitoring and the NAN results in a scalable architecture that can cope with future implementations and additions to the system.

This design is currently being implemented on University of Manchester campus. The ICT architecture for this project (Figure 1) is based on the network architecture described in more detail in (Pourmirza and Brooke, 2013). It contains a server side and a client side. The server side itself has 3 layers which are infrastructure layer, persistence layer, and application layer. The infrastructure layer is itself componentized into three monitoring levels, each relating to a specific section of the NAN in the distribution sub-Grid. The monitoring system implemented at the building level utilises smart meters, which are used to monitor the Home Area Network level data. These devices are located in all the buildings (our HAN level) in our campus test bed, transmitting data every 30 minutes. They are already connected to the power network and communicate by wired connections.

The monitoring system implemented at the street level is a wireless sensor network (WSN) which is used to monitor the environmental data such as temperature, light, and humidity, which are being logged every second. These environmental data are important for understanding and controlling the power grid since they can provide information that can be used to anticipate demand and improve control actions. These sensors run on batteries. The battery life with a $1 \%$ duty cycle would be 6 months (Kling, 2003). These sensors are able to alert when they run low on battery power. Since these sensors are grouped into clusters and have direct communication to their cluster head the routing will not be affected while changing the battery.

The final level of the monitoring is the substation level monitoring. The devices used for this level are reconfigurable real-time control and acquisition systems called compact RIOs. 16 cRIOs are located in each substation in our campus test bed logging data from the electrical network four times a second. They are connected to the power network and are able to transmit data through wired and wireless communication. Electrical network attributes such as three-phase voltage, current, active power, power factor can be monitored at this level which can be used for fault identification, power quality analysis, and many more applications.

At the moment the building level and the substation level metering devices are implemented in a real test bed, already producing live data. The street level monitoring devices are not available yet, thus we have used WSN simulation called TOSSIM to simulate the data at this level. The advantage of TOSSIM is that it enables the users to take their implementation and run it on an actual sensor when these are available. Thus we can test our prototype network in the laboratory based environment and also in a real physical environment. To achieve this, TinyDB (Madden et al., 2005) which is a WSN query processing engine, was extended to extract environmental data from the electrical Grid (Pourmirza and Brooke, 2012a). The difference between TinyDB and a traditional DB is that, instead of passively receiving and archiving data, we can also receive real-time data in response to our queries. The three monitoring levels discussed in the infrastructure level will transmit their data to the next layer of the architecture called the persistence layer.

The persistence layer contains a local data base which stores all the data received from the infrastructure layer, and a database connectivity module which use an interface to connect to the next layer which is the application layer. The backup strategy embedded in this level will enhance the preservation of the data. Moreover it will accommodate the ever-increasing volume of data 
produced by infrastructure layer.

The application layer is a NET application which is able to connect to the outside world, and send HTTP request and respond to the client side. It can use some component in case of emergency to send alerts to the engineers in the field and grid operator behind the screen in the control room.

On the client side we have used technologies such as ASPX, CSS, JavaScript, Ajax, and Google Maps API to visualise the collected data so it can be viewed by the grid operator.

According to our knowledge this is the first software architecture introduced for the NAN in the distribution sub-Grid which could integrate various level of monitoring from building level to the street level and finally the substation level, and apply monitoring and control over the collected data at such level.

In the next section we will describe how this visualisation tool operates.

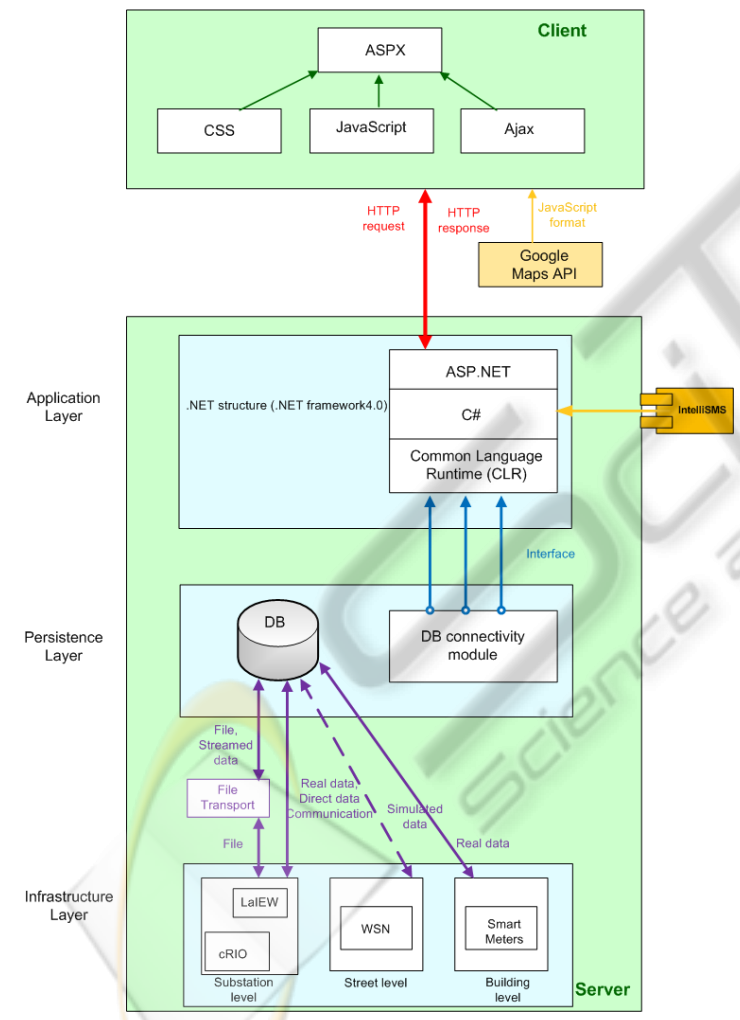

Figure 1: The developed software architecture.

\section{THE VISUALIZATION TOOL FOR A NAN}

While Smart Grid systems provide for automated control of the electrical networks, human operators are still essential players for certain monitoring and control tasks. We have developed a visualisation tool to enhance human understanding of performance of the electrical network. This tool operates at the application layer of our architecture and provides a basis for the development of Decision Support Tools for planning and operating the network.

\subsection{Related Work}

Visualisation is an eminent method for managing and displaying the data, which has been employed in different engineering fields. As an example in water distribution grids, visualisation has been used to provide a graphical user interface that enables the display of dynamic information and prediction of the future state of the grid. This information can be displayed via a Google maps based web interface (Haines et al., 2009, Stoianov et al., 2007). The Google map visualisation techniques can also be applied to other networked systems, such smart electrical grid.

A recent study by (Nga et al., 2012) proposed a visualisation technique exploiting Google maps and other techniques to display the data of the distribution network in the Smart Grid. The research mentioned above has provided a tool for monitoring the AMI and SCADA devices. These can only monitor and display the data of the critical areas of the distribution grid, whereas our proposed visualisation tool goes beyond that and can visualise the data from all over the distribution grid. Furthermore, our tool is able to visualise both the electrical grid data, and also environmental data from WSNs at street and building level. Thus all the data collected from the infrastructure layer can be visualised by our developed visualisation tool.

\subsection{A GIS-enhanced Visualisation Tool}

Our visualisation tool overlays the electrical grid and environmental information on a map using Google map API (Application Programming Interface). This research is being implemented on the medium voltage power network of the University of Manchester campus test bed, enabling the visualisation tool to be developed using real equipment, real data, and the feedback from professionals in the field. Figure 2 illustrates the additional functionalities added to the tool as first described in (Pourmirza and Brooke, 2012a) such as security authentication, fault detection and alarming 
system. Moreover, we have added visualisation of the data from the smart meters, deployed in our test bed, to the data collected from the cRIO devices in the substations. The figure below displays data over a period of one week. The $\mathrm{x}$-axis presents the time, here the days of a week, and y-axis represents the power consumed by the selected smart meter. The peak (week days) and off-peak (weekend) are illustrated.

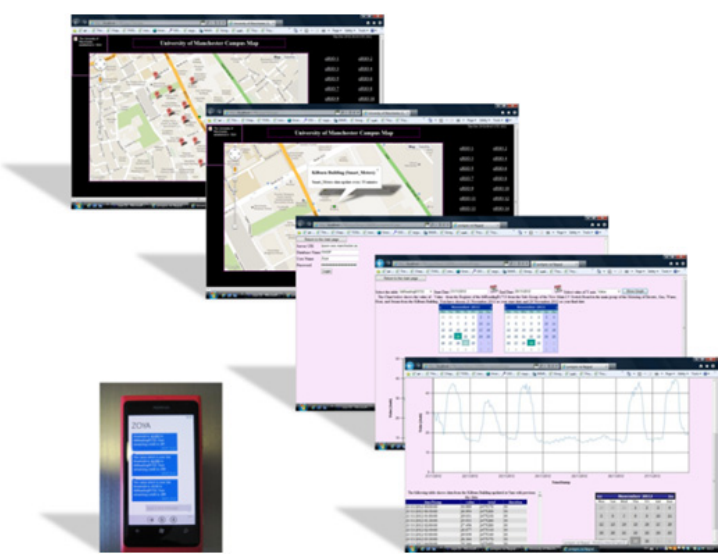

Figure 2: The developed visualisation tool.

\subsection{Data Flow Diagram}

The Figure 3 illustrates the level 1 Data Flow Diagram (DFD) which illustrates the flow of data and highlights the main functions carried out by the system.

When the application is started, the Google map GIS information will be loaded and the program will show the map view of our test bed. Then the user can follow three main actions. The main action is to select the desired metering device and send request for login to the database. After entering the correct login information the user can extract the data from the database and select the data table of interest for the desired period of time and then view the data in a new window. It can also plot the data graph. Showing the data on a data graph enhances better comprehension of the behaviour of the data. After visualising the data, it will determine if any of these data are over the defined threshold. If the threshold is exceeded then the tool will send an alert to any operators who have subscribed to it. For sending the alert it will follow two main actions. Firstly in order to send the passive alert it will highlight the over threshold data for the operator behind the screen. Secondly in order to send the active alert it will send text messages to the engineers in the field by using IntelliSMS component. It will inform them about the actual fault and time and location of it.
In addition there are two other flows that the user can follow. S/he can check the schematic view of the test bed and locate the desired metering devices on schematic map. The final flow is to start the TinyDB, in order to generate the environmental data and then send these data to the WSN simulator. Finally the user can select the WSN simulator on the visualisation tool and visualise the environmental data collected from the street level. All these collected data can then be used as an input for control algorithms of the grid.

\section{SUMMARY AND FUTURE WORK}

This research was undertaken to design an ICT architecture for the neighbourhood area of the Smart Grid (NAN), to analyse the various communication technology, and to find the most appropriate choices for this specific section of the grid. The present study justifies the need for having a sensor network alongside the power grid, and determines its possible applications in the future grid.

This paper described how we have designed and implemented a software architecture which is partially applied to the real test bed in the electrical network sub-Grids. We explain how we collect real data and how we use simulation to tune the implementation of the architecture on a real test bed. The main concern of this research is that it will enable information to be gathered from the working of a real system operating a sub-Grid level where monitoring has not previously been deployed.

The key contribution of our work is that it can deliver the information from the building levels, environmental conditions, and finally from the power grid below $33 \mathrm{kV}$ which remains unknown until now. According to our knowledge there is no previous architecture that could integrate all the information as a whole system for this level of the electrical Grid and present them via visualisation tool. This tool acts an essential component in operation and planning of the system.

In future, more work needs to be done to add Web Services to this architecture and to add more functionality to the visualisation tool. For example, automatically detecting the failure of nodes and alerting the field engineers. Finally, a further stage of this research is to investigate control strategies, for the NAN level of the Grid, to further the decentralised control of the whole Grid. 


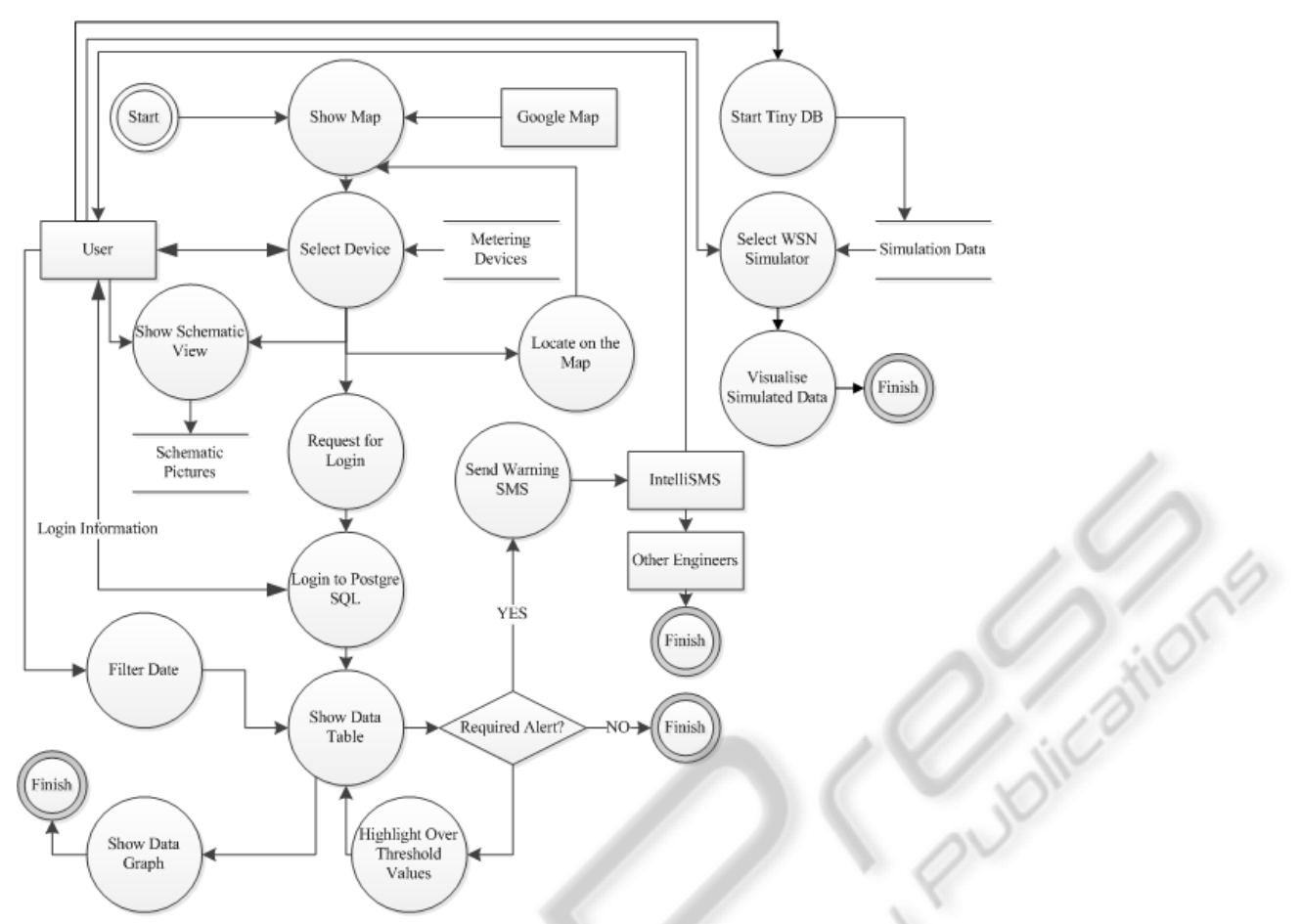

Figure 3: The DFD diagram.

\section{REFERENCES}

Haines, R., Khan, K. \& Brooke, J. 2009. Bringing simulation to engineers in the field: a Web 2.0 approach. The Royal Society, 2635-2644.

Kling, R. 2003. Intel Research mote. Santa Clara, USA: Intel Corporation Research.

Levis, P. \& Lee, N. 2003. TOSSIM: A Simulator for TinyOS Networks. University of Berkeley.

Levis, P., Madden, S., Polastre, J., Szewczyk, R., Whitehouse, K., Woo, A., Gay, D., Hill, J., Welsh, M., Brewer, E. \& Culler, D. 2005. TinyOS: An Operating System for Sensor Networks Ambient Intelligence. Springer Berlin Heidelberg.

Madden, S. R., Franklin, M. J., Hellerstein, J. M. \& Hong, W. 2005. TinyDB: An Acquisitional Query Processing System for Sensor Networks. ACM Transactions on Database Systems, 30, 122-173.

Nga, D. V., See, O. H., Quang, D. N., Xuen, C. Y. \& Chee, L. L. 2012. Visualization Techniques in Smart Grid. Smart Grid and Renewable Energy, 3, 175-185.

Pourmirza, Z. \& Brooke, J. 2012a. An Experimental Communication Architecture for Monitoring and Control of Sub-Grids. In: SMARTGREENS 2012 - 1st International Conference on Smart Grids and Green IT Systems, 2012a Porto, Portugal. SCITEPRESS Science and Technology Publications, 67-72.

Pourmirza, Z. \& Brooke, J. M. 2012b. The Monitoring Network Architecture in the Neighbourhood Area of the Smart Grid. Salford Postgraduate Annual Research
Conference (SPARC). Salford, UK.

Pourmirza, Z. \& Brooke, J. M. 2013. The Wireless Sensor Network and Local Computational Unit in the Neighbourhood Area Network of the Smart Grid. In: International Conference on Sensor Networks (SENSORNETS), 2013 Barcelona, Spain. SCITEPRESS - Science and Technology Publications, 84-88.

Stoianov, I., Nachman, L., Madden, S. \& Tokmouline, T. 2007. PIPENETa wireless sensor network for pipeline monitoring. In: Proceedings of the 6th international conference on Information processing in sensor networks, 2007 Cambridge, Massachusetts, USA. 1236396: ACM, 264-273. 